\title{
Evidence on the Comparison of Telephone and Internet Surveys for Respondent Recruitment
}

\author{
Dimitris Potoglou ${ }^{*}$, , Pavlos S. Kanaroglou ${ }^{2}$ and Neil Robinson ${ }^{1}$ \\ ${ }^{I}$ RAND Europe, Westbrook Centre, Milton Road, Cambridge, CB4 1YG, UK \\ ${ }^{2}$ Centre for Spatial Analysis (CSpA), School of Geography and Earth Sciences, McMaster University, Hamilton, \\ Ontario, L8S 4K1, Canada
}

\begin{abstract}
Internet surveys have a potential use for survey research when compared against costs and declining response rates of traditional modes as they form a powerful tool for reducing respondents' burden in complex questionnaires. On the other hand, there exists scepticism about the reliability and robustness of the collected data. Arenze et al. (2005) argue that case studies involving Internet surveys cannot be generalised to other countries and have recommended systematic collection and reporting of experiences worldwide. Such studies have had limited exposure in the transport literature. This paper provides empirical evidence on the comparison between telephone and Internet surveys in the context of a car ownership study. The comparison between telephone and Internet modes focuses on performance measures such as response speed, response rates, survey costs, demographic profiles and geographical representation of the sample. The results indicate the cost effectiveness of Internet surveys. Moreover, they show that the time and cost for data collection significantly vary by sampling and recruitment method. Finally, Internet survey response rates are lower than those in the telephone interview, which implies that Internet surveys can only be used to complement traditional data collection methods.
\end{abstract}

Keywords: Telephone surveys, internet surveys, survey comparison, car ownership, recruitment.

\section{INTRODUCTION}

Telephone surveys constitute traditional and reliable methods for data collection, and have been extensively used in transportation research. They offer key advantages relative to other methods including population representation, better resolution of respondent misunderstanding of questions and response [1]. Over the last decade, however, it has become more difficult to achieve contact when conducting telephone surveys. The main reasons include call-screening technology (e.g. answering machines, caller identification) and lack of participation associated with the increase in telemarketing. The recruitment of those who have cancelled subscription to landlines and have switched to mobile or voice over Internet (VOIP) technologies presents an additional challenge. These factors have contributed to increased costs of telephone surveys $[2,3]$.

On the other hand, the use of the Internet to collect survey data, particularly the World Wide Web, has grown significantly in the last fifteen years. An important reason for their popularity is the growing number of Internet users, rendering online survey techniques an ideal approach for the administration of surveys $[4,5]$. Internet surveys also permit more interactivity and realism, opening up the way for improved forms of representation. For example, the choice context in stated choice experiments has been enhanced with

*Address correspondence to this author at the RAND Europe, Westbrook Centre, Milton Road, Cambridge, CB4 1YG, UK;

Tel: +44 1223353 329; Fax: +44 1223358 845;

E-mail:dimitris@rand.org images, video, interface and functionalities of online markets for goods and services [6]. Moreover, the Internet and computerised interviewing, in general, provide more possibilities for personalising questions and automated routing through the survey.

To date, online surveys are more favourable than other modes due to their speed of implementation and cost effectiveness [7]. They are also more efficient from the perspective of data entry, quality and management [7]. For example, the self-administered nature of many forms of Internet-based surveys means that risks of misinterpretation by the interviewer are minimised as well as any room for errors and mistakes [8]. There are also benefits from the users' own perspective, in that they may feel more comfortable to present honest and unbiased answers due to the perceived anonymity of 'talking to a computer', though this benefit may depend on the privacy assurances made by the research team [9]. Finally, Internet surveys can provide a basis for data quality as the data are directly recorded into the survey analysis tool at the point of entry thus avoiding potential coding errors $[10,11]$. Due to the demographic characteristics of Internet users, such surveys have also been applauded as a means to reach busy professionals, a group for whom mail and telephone-based surveys are increasingly ineffective [12].

As with telephone and mail surveys at the time of their incorporation into the list of acceptable methodologies in earlier decades, Internet surveys are not without methodological concerns. One of the main criticisms levelled against Internet-based surveys is that they suffer from bias in the sampling frame, in respect of access to an Internet 
enabled computer being a prerequisite for respondents [13]. Although the proliferation of Internet cafés and mobile Internet-enabled devices is increasing, this is still a legitimate criticism, since the digital divide remains a reality $[14,15]$. Furthermore, despite efforts to achieve a balanced sampling frame, (for example, in nationally consistent studies) data illustrates that Internet surveys obtain responses from younger, highly educated and high-income individuals $[16,17]$. A second major concern with Internet-based surveys is the question of usability [18]. This is particularly important as those administering Internet-based surveys must take into account that the survey instrument may be viewed on a very heterogeneous environment of computer, email and Internet-browser configurations, with varying screen resolutions and character-encodings. Achieving maximum usability across this environment is a complex task. Other issues that may affect responses include anxiety or uncertainty regarding the use of a computer generally, and concern and fear over security and privacy $[13,18]$. Finally, respondent recruitment through non-probability panels is considered another area of concern regarding Internet surveys [19].

Given that the ultimate goal of every study is to collect high quality data, response speed, response rates and data collection costs constitute important design trade-offs [12]. Our review of the literature yields a very small number of studies that directly compare telephone interviews and Internet surveys. The purpose of this paper is therefore to compare response speeds, response rates, costs and demographic profiles of respondents between Internet-based surveys and telephone interviews after recruiting respondents through convenience and probability sampling. Comparisons are based on an empirical study that involved two in-parallel data collection campaigns in the context of recruiting participants in a stated choice experiment on alternative fuelled vehicles in the Census Metropolitan Area of Hamilton, Canada.

\section{INTERNET $V S$ TRADITIONAL METHODS FOR TRANSPORTATION ANALYSIS}

A range of evaluations of Internet-based surveys have been conducted as these surveys become popular and viable alternatives to self-completed mail questionnaires and computer-assisted telephone interviewing (CATI) modes [20]. However, due to relatively recent use of Internet-based surveys and the high costs associated with their evaluations, there has been little comparative research between traditional survey techniques and the Internet.

In the broader survey literature, comparison studies between the Internet and traditional modes of data collection include Cobanoglu et al. [21], Crawford et al. [22], Fricker et al. [23], and Braunsberger et al. [10].

Cobanoglu et al. [21] compare mail, fax and web-based surveys in terms of response speed, response rate and cost in the US university-setting, using a sample of 300 professors. The authors report that the fastest methods are fax and web with an average response time of 4 and 6 days, respectively. The slowest mode is mail with an average of 16.5 days to respond. The web survey achieves the highest response rate (44\%) followed by mail (26.3\%) and fax (17\%). The authors recommend mixed-mode surveys using e-mail/web when surveying educators. Crawford et al. [22] compare Internet and mail surveys by recruiting university students as part of a study on drug and alcohol use. The web-survey achieves $20 \%$ higher response rate than the mail counterpart. The costs by mode show that a web-based data collection facility with established infrastructure is financially more efficient than a mail survey. Fricker et al. [23] compare telephone with Internet surveys for respondents who have an Internet connection and are initially recruited via random digit dialing. The authors report that Internet respondents are fewer than those who respond over the telephone, in spite of the fact that Internet respondents are offered higher incentives. The Internet survey produces less item nonresponse than the telephone survey. Also, Internet respondents take longer to complete the knowledge items, particularly those requiring open-ended answers than the telephone respondents. Finally, Braunsberger et al. [10] compare telephone with Internet surveys. Web respondents are members of an Internet panel whereas the telephone survey uses a 'cold-calling' method to randomly selected respondents. The results show that while web panels might not be appropriate for all survey research endeavours, they could be a viable alternative to telephone surveys that allows researchers to conduct high quality research.

The first two comparison studies focus on specific sampling frames (i.e., students, university faculty), thus making it difficult to generalise the results in other contexts and disciplines. Fricker et al. [23] and Braunsberger et al. [10] employ slightly different ways of recruiting respondents than the present study. Fricker et al. [23] only recruit participants connected to the Internet whereas Braunsberger et al. [10] recruit participants via online panels. Researchers are therefore likely to develop a variety of ways in recruiting and surveying respondents using traditional and online methods.

Transport surveys, in particular, pose additional challenges as they aim at collecting larger samples and involve additional complexities such as recruiting and surveying respondents in several stages (e.g. stated choice surveys, travel diary surveys). Comparisons, however, between Internet and traditional surveys for transportation analysis and travel behaviour research are rare. Arentze et al. [24] report experiences with the use of Internet surveys using three case studies of varying complexity. Their findings suggest that Internet surveys offer potential in administering complex choice tasks such as stated preference and stated adaptation experiments. Moreover, Internet surveys prove a perfect match for interactive experiments where controls are required on the input data and the survey involves data collection on how individuals respond to new information. At the same time, Arentze et al. [24] stress that unavailability of a sampling frame requires recruitment of participants through various announcements, which may cause subsequent biases, thus calling into question the representativeness of the sample against the population of interest. In addition, even in the case where a sampling frame is available, there is still the risk of under-representing population segments such as the elderly and women [24]. Finally, the authors recommend that experiences with Internet-based surveys in other countries be reported as case studies since results are more likely to differ because of 
differences in Internet penetration and history of Internet use.

In the search for a reliable survey protocol, given time and budget constraints, Bayart and Bonnel [25] compare web and face-to-face household-travel surveys in the city of Lyon, France. The authors find that Internet respondents are more often executives and employed persons with higher income and levels of motorisation compared with participants in the face-to-face survey. Moreover, Bayart and Bonnel [25] report that Internet respondents spend less time at home, making them less likely to be recruited through traditional survey modes. Similar trends are reported by de Blaeij et al. [26], who, in a study that focuses on e-shopping and in-store shopping behaviour of residents in Utrecht and its surrounding cities in the Netherlands, find statistically significant self-selection effects in the composition of the Internet sample over a mail sample. Again, males, individuals with high income and previous Internet and eshopping experience are the most important factors driving preferences to complete Internet surveys over mail surveys. Residential location is also significant; individuals residing in the city centre are more likely to complete the Internet survey than those living in rural areas. However, against $a$ priori expectations, de Blaeij et al. [26] report that the elderly are more likely to participate in the Internet survey. The comparison between Internet and mail responses is conducted on the basis of socio-demographic, personality, Internet- and experienced time pressure variables, as well as the e-shopping behaviour of respondents. Blaeij et al. [26] find no differences in the quality of the data and the level of non-response between Internet and mail surveys. Finally, Mix et al. [27] compare samples of respondents working in the downtown area of Buffalo, New York, obtained through Internet and mail surveys. Their findings suggest that while cost, time and response in the Internet survey are significantly less than the mail survey sample, data between the two modes are comparable, if not identical.
Overall, little empirical evidence exists on how Internet surveys compare with traditional modes. There is often lack of consistency across comparisons as those follow different recruitment and survey implementation approaches. Since results are more likely to differ across studies and contexts, the aim of this paper is to report on the experience from a case study conducted in a metropolitan area with high internet penetration and extended history of internet use. In particular, the study aims at obtaining a better understanding of using the Internet for survey data collection when using different sampling approaches - i.e., probability based and convenience sampling. It further assesses differences across modes (and sampling approaches) in terms of response times, costs and characteristics of respondents.

\section{BACKGROUND AND DESIGN OF THE STUDY}

\subsection{Structure of the Questionnaire}

As shown in Fig. (1), the survey questionnaire was comprised of two stages. Stage 1 was employed to recruit participants and collect background information and Stage 2 involved a stated choice experiment. The comparisons in this paper focus on Stage 1, which included a total of 107 questions organised into six sections [28]:

Household information: number of household members, lifecycle stage of the household, total household income, number of vehicles, household location, home-ownership status and number of years lived at the current dwelling,

Personal characteristics including age, gender, educational attainment, employment status, race/ethnicity and driving license-holding status,

Household history information: date the household was formed and total time its members have lived in the study area,

Type and vintage of vehicles owned or leased and number of vehicles previously bought sold and/or disposed,

\begin{tabular}{|l|c|c|c|}
\hline & Survey A & \multicolumn{2}{|c|}{ Survey B } \\
\hline Sampling Method & Non-probability & \multicolumn{2}{|c|}{ Probability } \\
\hline Survey Mode & Internet & $\begin{array}{c}\text { Survey B1 } \\
\text { Phone/Mail-Back }\end{array}$ & \multicolumn{2}{|c|}{ Survey B2 } \\
Internet
\end{tabular}

Fig. (1). Structure of surveys (Source: Potoglou, 2006). 

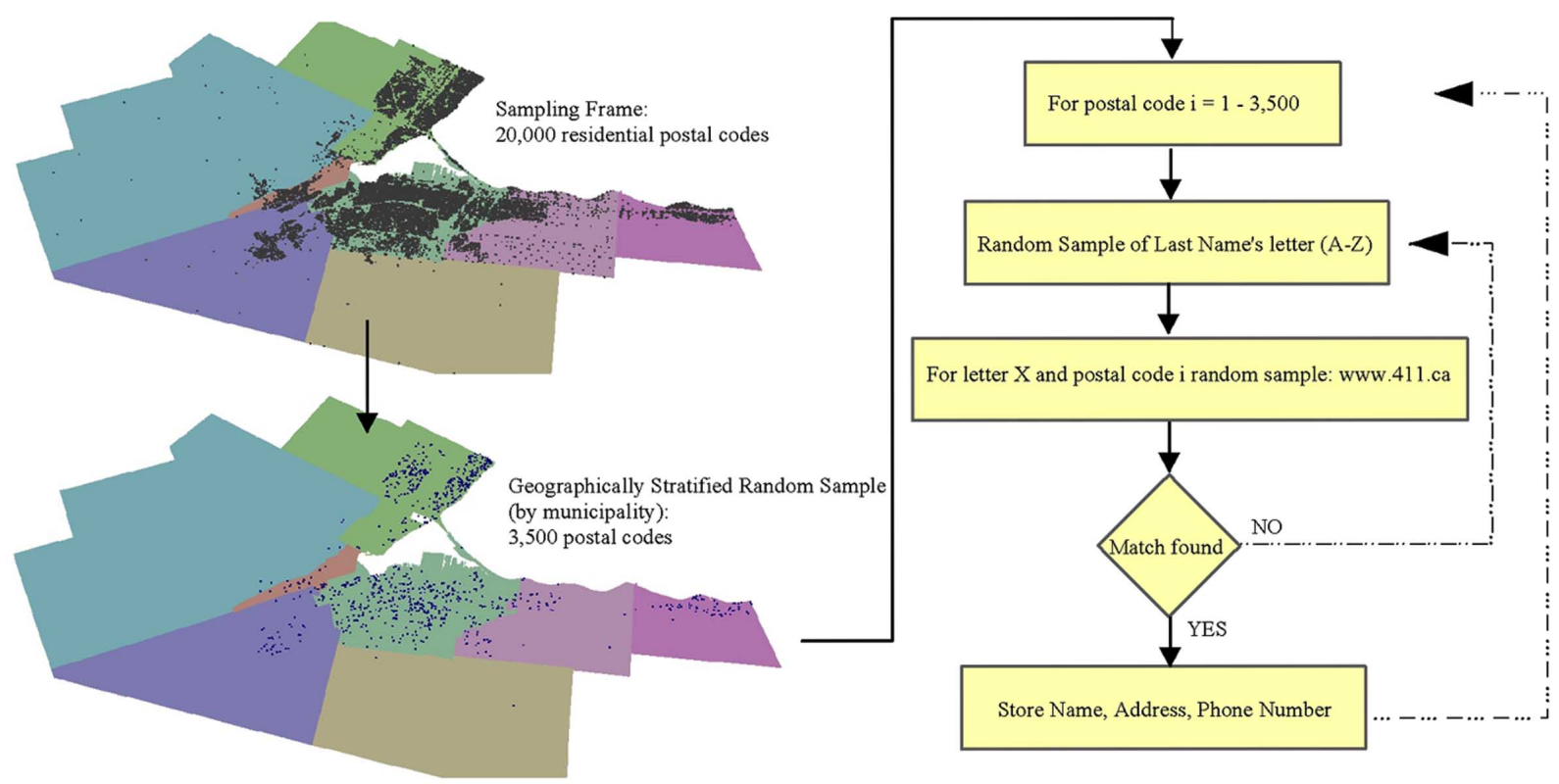

Fig. (2). Sampling procedure in Survey A.

Information on household's history such as the time people joined or left the household,

Future plans to purchase a vehicle including details about its characteristics such as size, anticipated annual usage rate and purchase cost.

\subsection{Design and Administration of Surveys}

The questionnaire was developed as an Internet-based application package using Hyper Text Markup Language (HTML) and Personal Homepage Page (PHP) language. A number of programming tasks allowed automatic coding of vehicle information through integration with the Fuel Economy Guide database [29]. Fuel economy, size and annual fuel cost, were automatically integrated into the database based on participant's current vehicle make and model. The application package could also be used as a computerised-telephone-interview tool.

Prior to the launch of the main surveys, a pre-test and two pilot surveys were employed to improve several technical and cognitive issues of the instrument.

\section{Survey A: Telephone and Internet Surveys Using Geographically Stratified Random Sampling}

As shown in Fig. (2), potential respondents in Survey A were drawn from a two-stage sampling design. At the first stage, a geographically stratified random sample of 3,500 postal codes was drawn without replacement. The strata corresponded to the eight municipal districts of the Hamilton area. At the second stage, we randomly sampled and matched households' contact information for each sampled post code using an electronic telephone directory. As shown in Fig. (2), the iterative procedure for randomly sampling the contact information of potential respondents given a randomly sampled postcode ensured a $100 \%$ match rate .

\footnotetext{
${ }^{1}$ For example, if the sampled postcode has no match to a given letter corresponding to a respondent's last name, the algorithm would randomly re-sample another letter and search for another match. The algorithm
}

Participants were invited to the survey through a personalised letter a week before the telephone contact was made. The letter also provided respondents with the Internet link and a personal identification number (PIN) in case they preferred to complete the survey on the Internet.

The telephone interview was conducted using the control panel in Fig. (3). The interface of the panel allowed the interviewer to retrieve participants' interview status and perform a set of tasks including the telephone interview.

The control panel was also programmed to make a total of four attempts to establish contact with respondents at different days and times in case of a busy tone, an answering machine or no answer at the first attempt. No further attempt was made when: the phone was disconnected, the household was located outside the study area, respondents refused to participate, did not speak English or stated that they would complete the survey on the Internet.

The telephone interviews were conducted between March 2005 and May 2006. The majority of the telephone interviews were conducted in the evenings of weekdays between 5:00 pm and 9:00 pm and on Saturdays between 12:00 pm and 5:00 pm by three interviewers. Participants were eligible for 13 monetary prizes worth a total of C $\$ 900$. The average completion time of the phone interview was 30 minutes.

\section{Survey B: Internet Survey Using Convenience Sampling}

Survey B was only available on the Internet ${ }^{2}$. Recruitment of potential respondents was undertaken in several ways. Prior to the official release of the survey, we contacted managers and human resources administrators of major employers in the area such as the City of Hamilton ( 7,500 employees), McMaster University $(\sim 3,500$ employees) and Hamilton Health Sciences $(\sim 8,000$

continues until a match between postcode and contact information is successful.

${ }^{2}$ The survey is available for testing at http://www.science.mcmaster.ca/ pot ogld/php/web/index2 


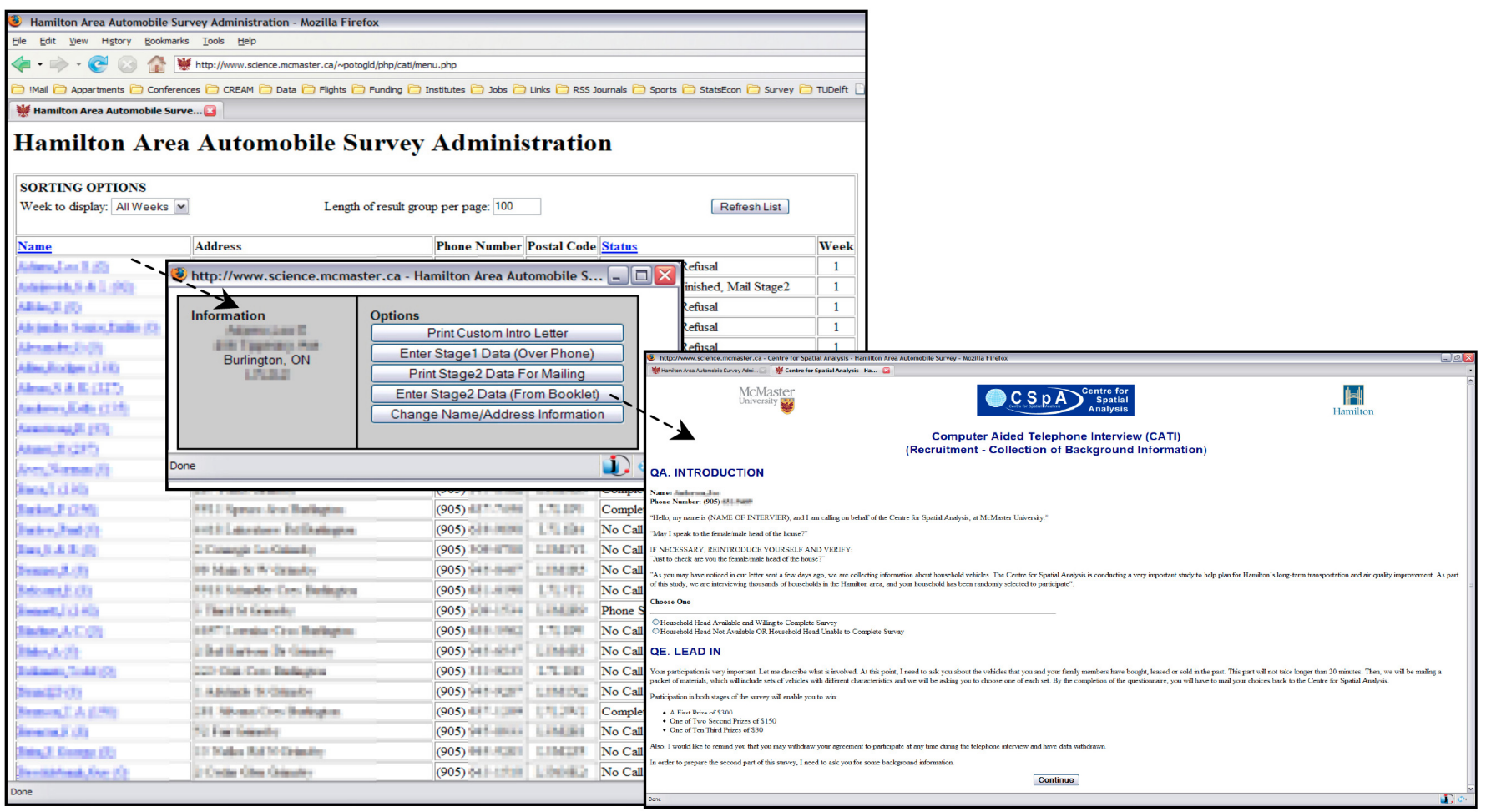

Fig. (3). Control panel for telephone interviews.

employees) requesting to host a notice about the survey on their Intranets or distribute the e-mail invitation to their employees. Recruitment of respondents also relied on snowballing. Finally, public announcements were placed on the local university website and newspaper.

Survey B was conducted in April 2005 [30]. Following the first e-mail invitation, a total of four reminders were sent during the second, fourth and fifth weeks. Respondents accessed the survey by first providing their e-mail address, which was also used to control for multiple entries. Upon recording a respondent's e-mail address, a PIN number and a link to the questionnaire were automatically sent to a participant's e-mail address. This procedure allowed respondents to complete the survey in multiple steps at different times ${ }^{3}$. Furthermore, it reduced the risk of partially completed questionnaires due to accidental exits from the browser, electric-power failure or respondents' fatigue. Similarly with Survey A, incentives included monetary prizes with a total worth of C\$600.

\section{EVALUATION OF SURVEYS}

\subsection{Response Speed}

Survey B was by far the fastest method of data collection with an average of 23 completed responses per day (894 respondents over six weeks), whereas the average response speed in Survey A (phone and Internet combined) was 16 questionnaires per week ${ }^{4}$.

\footnotetext{
${ }^{3}$ The average completion time for respondents who completed the survey in one step was 15 minutes.

${ }^{4}$ Survey A1 (phone interviews) alone reached an average of 12 responses per week whereas the response-speed of a probability-based Internet survey (Survey A2) was 4 responses per week.
}

Compatibility between the recruitment method and surveying modes may have affected response speeds. The Internet-based response speed was very high when respondents were recruited via electronic means such as intranets and e-mail (Survey B). On the other hand, the response speed of the Internet-survey was lower when respondents were invited to participate via a hardcopyadvance letter (Survey A2).

Fig. (4) shows the response patterns of Surveys A and B and provides empirical evidence into the seasonal variation of responses, which is in line with previous studies [e.g. 31]. Specifically, response speed in the case of Survey B is high and appears as a continuous curve within the six weeks of data collection. Responses in Survey A, on the other hand, appear discontinuous because of very poor response rates observed during the summer months and Christmas time in 2005.

\subsection{Response Rates}

The advance letter of recruitment is considered an essential element of the tailored design method [7]. Of all advance letters sent to each of the 3,500 households in the sampling frame of Survey A, only 197 (5.6\%) of those letters were returned as undeliverable, which is indicative of the good quality of contact information data. Table 1 shows all possible results of the interviewing procedure.

The overall response rate of Survey A (using a probability-based sample) reached $29.3 \%$, which is considered a fairly good response rate given the complex, retrospective nature and length of the questionnaire The majority of participants in Survey A opted for the telephone interview $(23 \%)$, and only $6.3 \%$ of respondents participated via the Internet, which indicates the difficulty of obtaining 


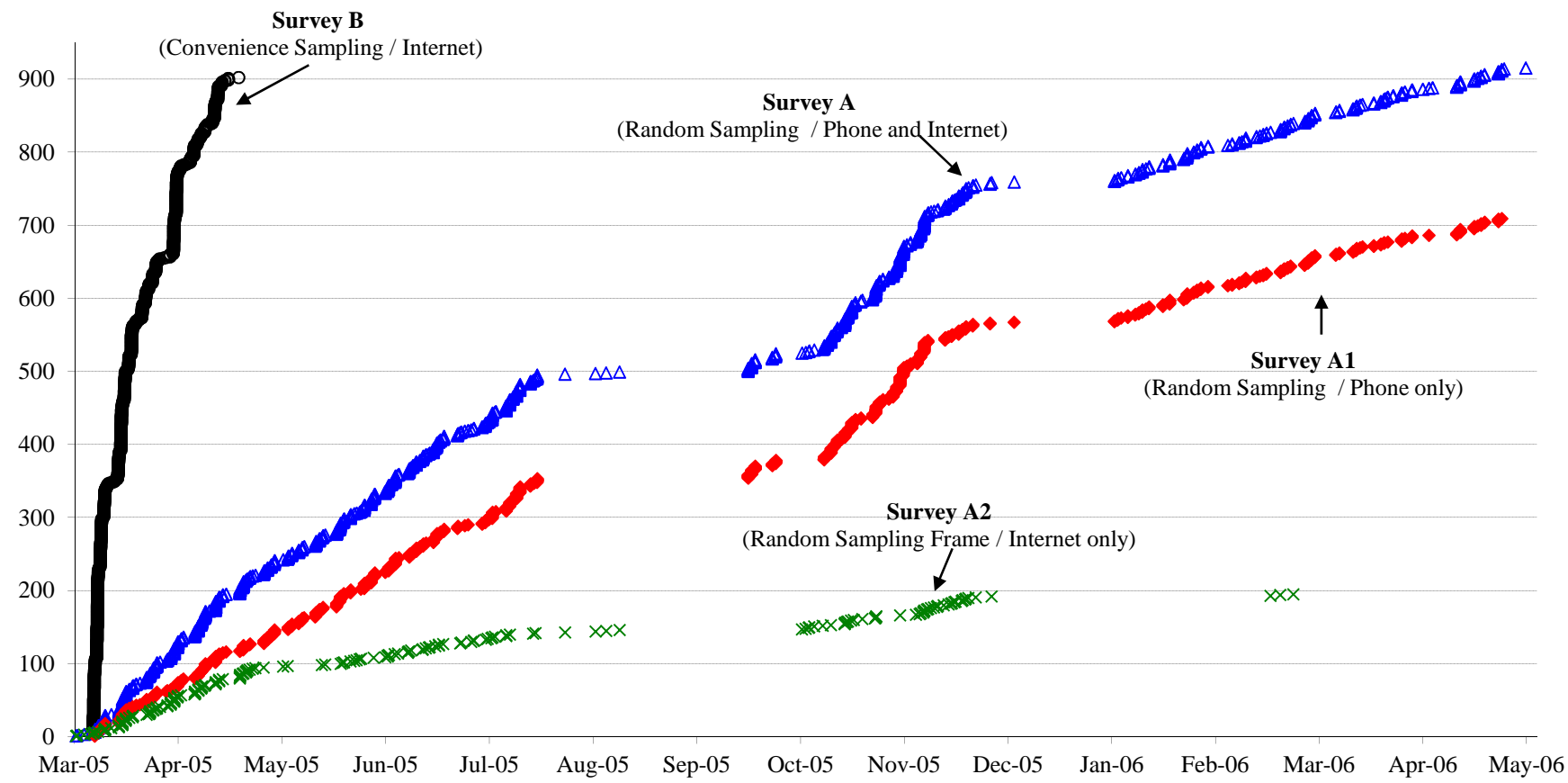

Fig. (4). Response patterns.

Table 1. Distribution of Interview Outcomes in Survey A

\begin{tabular}{|c|c|c|c|}
\hline & Nr. of Households & Raw Sample (\%) & Adjusted Sample ${ }^{5}(\%)$ \\
\hline \multicolumn{4}{|l|}{ Interviewing Result } \\
\hline Completed $^{6}$ by phone-interview (A1) & 713 & 20.9 & 23.0 \\
\hline Completed via the Internet (A2) & 201 & 5.7 & 6.3 \\
\hline Refused & 1206 & 34.5 & 37.9 \\
\hline Stated they would complete via the Internet, but did not & 205 & 5.9 & 6.4 \\
\hline Four or more calls with no answer & 644 & 18.4 & 20.2 \\
\hline Answering machine (four or more calls) & 52 & 1.5 & 1.6 \\
\hline Number not in service & 218 & 6.2 & - \\
\hline Not in sample (non residential or out of the area of study) & 97 & 2.8 & - \\
\hline Privacy service & 44 & 1.3 & 1.4 \\
\hline Language barrier & 102 & 2.9 & 3.2 \\
\hline Total & 3,500 & 100.0 & 100.0 \\
\hline
\end{tabular}

Internet responses when potential participants are recruited via non-electronic means.

Response rates in Survey B cannot be computed because there are no explicit sampling frames since it is unknown how many individuals may have seen the survey or links to the survey, but decided not to participate. Therefore, response rates for Survey B are not meaningful and cannot be interpreted in the same way as response rates in probability samples $[32,33]$.

\footnotetext{
${ }^{5}$ Adjusted Sample $=$ Sample Size $-($ Number not in service + Not in sample)
} ${ }^{6}$ Respondents provided answers to all stages of the questionnaire

\subsection{Survey Costs}

Table 2 presents fixed and variable costs in Surveys A and B. Before adding the influence of the differential response rates or redemption of incentive money-prizes, the overall cost of fielding Survey A was approximately $50 \%$ higher than the overall cost of fielding Survey B (the Internet survey using a convenience sample) to the same number of respondents.

Fixed costs in Survey A included the design of the survey, software development, purchasing contact information and handling and posting the introduction letters to potential respondents. Fixed costs incurred in Survey A 
Table 2. Summary of Costs of Internet and Phone/Mail-Back/Internet

\begin{tabular}{|c|c|c|c|}
\hline Response Mode & $\begin{array}{c}\text { Phone } \\
\text { (Survey A1) }\end{array}$ & $\begin{array}{c}\text { Internet } \\
\text { (Survey A2) }\end{array}$ & Internet \\
\hline \multicolumn{4}{|l|}{ Fixed Costs } \\
\hline Survey Design \& Software Development & $\mathrm{C} \$ 5,000$ & $\mathrm{C} \$ 5,000$ & $\$ 5,000$ \\
\hline Advance Letters & $\mathrm{C} \$ 4,025$ & $\mathrm{C} \$ 4,025$ & - \\
\hline Variable Costs & $\mathrm{C} \$ 14,000$ & - & - \\
\hline \multirow{2}{*}{\multicolumn{4}{|c|}{$\begin{array}{l}\text { Phone Recruiting \& Interviews } \\
(774 \text { hrs. } * \$ 18 / h r)\end{array}$}} \\
\hline & & & \\
\hline Total Costs & $\mathrm{C} \$ 24,035$ & $\mathrm{C} \$ 10,035$ & $\mathrm{C} \$ 5,600$ \\
\hline
\end{tabular}

Note: All amounts are in 2005 Canadian dollars.

are also listed for both Surveys A1 and Survey A2 only for comparison purposes, and in order to allow for relative comparisons of the "per unit" cost across response modes (i.e., phone, Internet).

Overall, the Internet survey using convenience sampling (Survey B) was the least expensive $(\mathrm{C} \$ 5,600)$, whereas Survey A cost approximately seven times more (C\$34,070). With regard to Survey A, the cost of data collection using probability sampling and phone interviews (Survey A1) was C $\$ 24,035$ and the cost of collecting data using probability sampling and the Internet (Survey A2) was C\$10,035. The reported "per unit costs" are dependent on the response rates achieved in each survey, and cannot be generalised. However, given the relatively high response rate (approx. $30 \%$ ) and the low level of preference for the Internet option in Survey A, the reported costs provide a good indication for comparison of the overall costs involved in each survey. Also, it is shown that there is a notable difference between phone interviews and Internet surveys depending on the type of sampling and recruitment methods.

\section{DATA QUALITY: INTERNET VS PHONE SAMPLES}

\subsection{Geographical Representation}

Fig. (5) shows the spatial distribution of respondents' place of residence in the retrieved samples. For comparison purposes, the samples are contrasted with the postcodes layer in the area (grey dots) and the distribution of the population based on the 2001 Canadian Census.

The sample in Survey A over-represented the areas of Burlington, Dundas, Stoney Creek and Grimbsy, whereas it under-represented the areas of Flamborough, Ancaster, Glanbrook and Hamilton. Moreover, higher numbers of Internet responses (Survey A2) came from the urbanised areas of Burlington and Hamilton followed by Stoney Creek and Grimsby. Responses in Survey B over-represented households in Hamilton, Ancaster, Flamborough and Stoney Creek, whereas it under-represents Burlington and Grimbsy. The estimated values of $\chi_{\text {Census-SurveyA }}^{2}=137,501$ and $\chi_{\text {Census- }}^{2}$
SurveyB $=109,441(d . f .=7)$ show that the difference between the two samples and the 2001 Canadian Census is statistically significant at the $99 \%$ level.

\subsection{Demographic Profiles}

Tables 3 and $\mathbf{4}$ present the demographic profiles in the collected samples. The first two columns in Table $\mathbf{3}$ show the sample composition in Survey A by survey mode, and the next two columns compare the totals obtained in Surveys A and $\mathrm{B}$, respectively. Comparisons across the sub-samples A1 and $\mathrm{A} 2$ reveal interesting differences in the characteristics of the phone and Internet respondents. Specifically, more males $(59.5 \%)$ than females participated in the Internet survey A2, whereas significantly more females $(50.1 \%)$ than males participated in the phone interviews $\left(\chi^{2}=35.23, d . f=1\right.$, $p=0.000)$. The majority of voluntary respondents in Survey B were females $(58.3 \%)$, which is in line with the general notion that females are more likely to participate in surveys $[7,33]$.

The age distribution across the sub-samples A1 and A2 are similar for individuals between 25 and 54 years of age, whereas the percentage of individuals between 55 and 64 years who participated via the Internet is higher than those who participated in the phone interview. The age distribution between the two subsamples is statistically different $\left(\chi^{2}=14.53\right.$, d.f. $\left.=6, p=0.024\right)$. Table 3 also shows that the Internet survey A2 included approximately equal proportions of all age groups of respondents between 35 and 65+ years of age, which implies that the Internet survey was userfriendly and accessible across age groups. On the other hand, the majority of deliberate responses via the Internet (Survey B) came from younger individuals between 25 and 54 years of age, whereas elderly households had little participation. This pattern was expected as recruitment of respondents was conducted mainly at their place of work.

The majority of responses (approx. 58\%) in Survey B came from highly educated individuals who held a bachelors degree or higher. By contrast, Survey A included higher proportions of respondents with lower education levels (high-school graduates or lower). The proportion of 


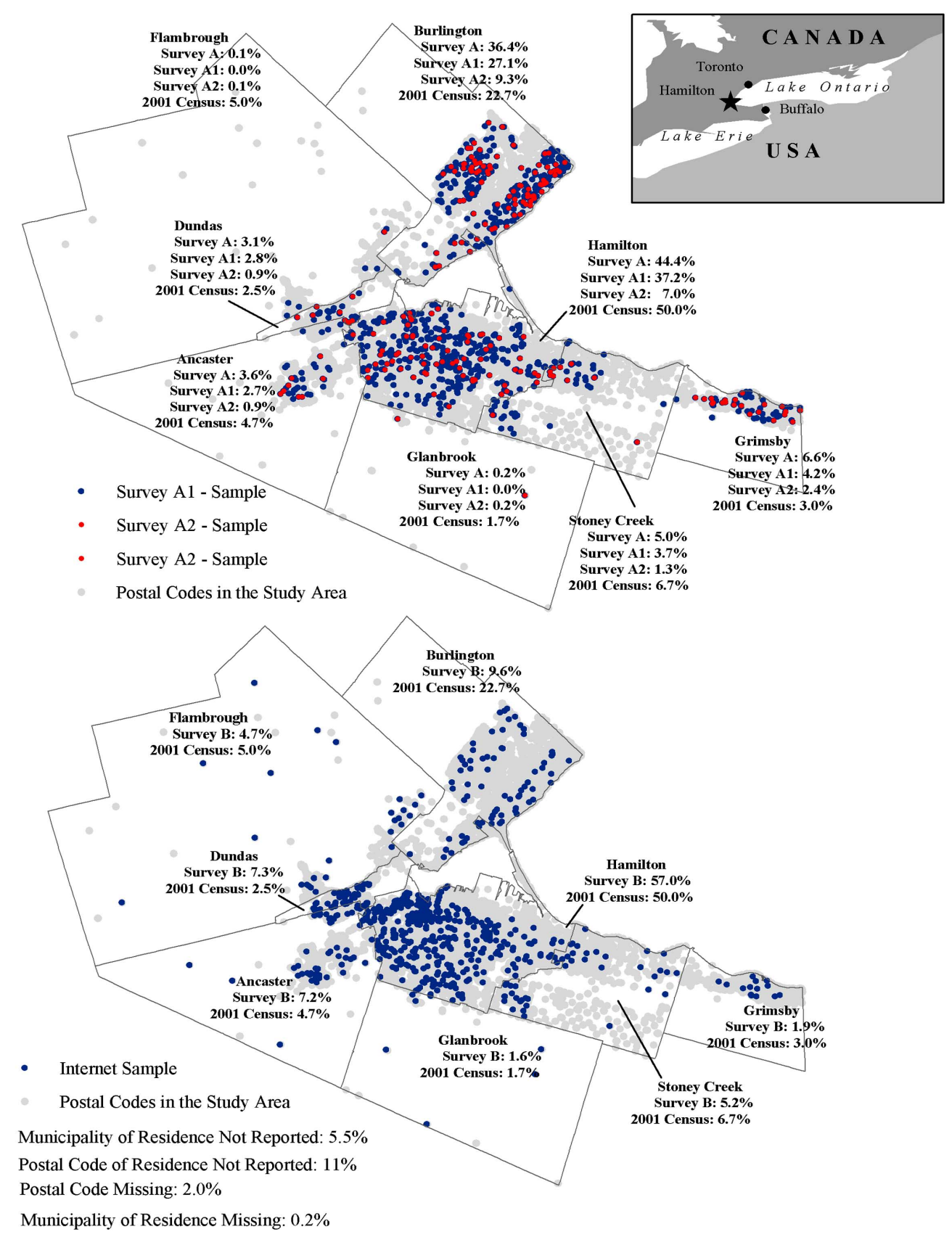

Fig. (5). Geographical representation and distribution of respondents.

respondents with the highest level of education in the Internet survey (A2) sample was more than double the proportion of those who participated in the phone interview. Relevant chi-square tests of independence show that the samples were significantly different in terms of education level and employment status. We further find that the proportions of the race/ethnicity variables were not statistically different between Internet participants in surveys $\mathrm{A} 2$ and $\mathrm{B}$, respectively $\left(\chi^{2}=1.59\right.$, d.f. $\left.=2, p=0.954\right)$.

Table 4 presents sample comparisons across household characteristics such as the number of household members, number of children and vehicles. The Internet samples A2 and $B$ were not statistically different in terms of the number of household-member structure $\left(\chi^{2}=1.14\right.$, d.f. $\left.=3, p=0.767\right)$, number of children $\left(\chi^{2}=6.85\right.$, d.f. $\left.=4, p=0.063\right)$ and number of vehicles in the household $\left(\chi^{2}=5.35, d . f .=4, p=0.253\right)$. Samples A and B awee not significantly different in terms of the number of household-members variable $\left(\chi^{2}=2.53, d . f=3\right.$, $p=0.456$ ) but they are significantly different regarding the remaining variables and overall when both are compared against the 2001 Census.

The Survey B sample included higher proportions of renters and individuals living in apartments or condos than Survey A, in which more than $90 \%$ of the respondents lived in a single family home. More than $25 \%$ of households in Survey A and $35 \%$ of households in Survey B earned more than C\$70,000 annually. However, Survey A included higher proportions of participants who do not report their income 
Table 3. Respondent Profiles (Sample \%)

\begin{tabular}{|c|c|c|c|c|c|}
\hline & \multicolumn{2}{|c|}{ Survey A } & \multirow{2}{*}{$\begin{array}{c}\text { Total } \\
\text { Survey A } \\
(n=915)\end{array}$} & \multirow{2}{*}{$\begin{array}{c}\text { Total } \\
\text { Survey B } \\
(n=894)\end{array}$} & \multirow{2}{*}{$\begin{array}{c}2001 \\
\text { Canadian } \\
\text { Census }\end{array}$} \\
\hline & $\begin{array}{c}\text { Survey A1 } \\
(\mathrm{n}=713)\end{array}$ & $\begin{array}{c}\text { Survey A2 } \\
(\mathrm{n}=\mathbf{2 0 2})\end{array}$ & & & \\
\hline \multicolumn{6}{|l|}{ Gender } \\
\hline Females & 50.1 & 39.0 & 47.7 & 58.3 & 48.8 \\
\hline Males & 49.2 & 59.5 & 51.4 & 40.4 & 51.2 \\
\hline No Response & 0.7 & 1.5 & 0.9 & 1.3 & - \\
\hline \multicolumn{6}{|l|}{ Age } \\
\hline$<24$ & 3.1 & 2.5 & 3.0 & 9.2 & 12.9 \\
\hline $25-34$ & 11.8 & 11.2 & 11.7 & 30.3 & 13.2 \\
\hline $35-44$ & 21.6 & 22.4 & 21.8 & 23.7 & 16.6 \\
\hline $45-54$ & 22.7 & 22.4 & 22.6 & 24.1 & 14.1 \\
\hline $55-64$ & 17.3 & 21.0 & 18.1 & 10.0 & 9.6 \\
\hline $65+$ & 22.0 & 18.1 & 21.1 & 1.5 & 14.3 \\
\hline No Response & 1.5 & 2.4 & 1.7 & 1.2 & - \\
\hline \multicolumn{6}{|l|}{ Education } \\
\hline High school or lower & 35.2 & 32.7 & 34.6 & 13.8 & 41.2 \\
\hline Diploma & 27.7 & 28.8 & 28.0 & 22.5 & 36.2 \\
\hline Bachelor's degree & 28.7 & 21.0 & 27.0 & 32.8 & 22.5 \\
\hline Graduate school & 6.4 & 13.2 & 7.9 & 25.8 & - \\
\hline No Response & 2.0 & 4.4 & 2.5 & 5.1 & \\
\hline \multicolumn{6}{|l|}{ Race/Ethnicity } \\
\hline Caucasian & 84.5 & 88.8 & 85.5 & 85.7 & 80.3 \\
\hline Non-Caucasian & 4.9 & 6.8 & 5.3 & 8.6 & 19.7 \\
\hline No Response & 10.6 & 4.4 & 9.2 & 5.7 & - \\
\hline \multicolumn{6}{|l|}{ Employment Status } \\
\hline Full-time & 53.3 & 56.1 & 53.9 & 76.6 & N/A \\
\hline Part-time & 9.5 & 9.3 & 9.4 & 12.6 & \\
\hline Unemployed & 4.0 & 3.9 & 4.0 & 6.5 & \\
\hline Retired & 28.7 & 25.4 & 28.0 & 2.0 & \\
\hline Full-time homemaker & 3.5 & 2.9 & 3.4 & 0.3 & \\
\hline No Response & 1.0 & 2.4 & 1.3 & 2.0 & \\
\hline
\end{tabular}

than Survey B (Survey A $=63.1 \%$ vs Survey B $=41.4 \%$ ). Compared with the phone-interview sample, the Internet surveys achieved lower proportions of unreported income.

\section{CONCLUSION}

While implementation of Internet-based surveys has been growing during the last decade, there exists little evidence on their efficiency relative to traditional modes of data collection such as computer aided telephone interviews. This paper presents findings from a comparison between phone interviews and Internet-based surveys, using both convenience and probability sampling. The results indicate that Internet-based surveys have significant advantages over phone interviews in terms of costs and response speed (see Table 5). However, Internet-based surveys are very much dependent on the method and mode of recruitment. In particular, when recruitment is conducted via convenience sampling and technology-based media (e.g. Intranets, Internet sites, e-mail lists) the response speed is extremely high, resulting in very low costs. On the other hand, response speed is slower and costs are higher than phone interviews when recruitment of respondents is conducted through a non- technology medium - i.e. advance letters sent by post. In spite of the advantages of Internet-surveys using convenience sampling, there are significant implications of sample quality and researchers should adopt such strategies with caution.

In terms of response rate, the findings of this study show that telephone interviews perform better than the Internetbased questionnaire when respondents are given the option to choose between the two modes. Overall, the combination of the two modes improves the response rate of the survey, which implies that Internet-based surveys can be used as a complementary mode to traditional methods. This strategy would also help to address problems of Internet coverage of the general population and the difficulty of drawing probability samples as discussed by Couper et al. [34]. Nevertheless, a carefully designed survey using convenience sampling may achieve a relatively high number of responses when the study requires responses from specific segments of the population and data must be collected within a short timeframe. The above findings are also in agreement with Dillman [7]. 
Table 4. Household Profiles ( $\%$ in the Sample)

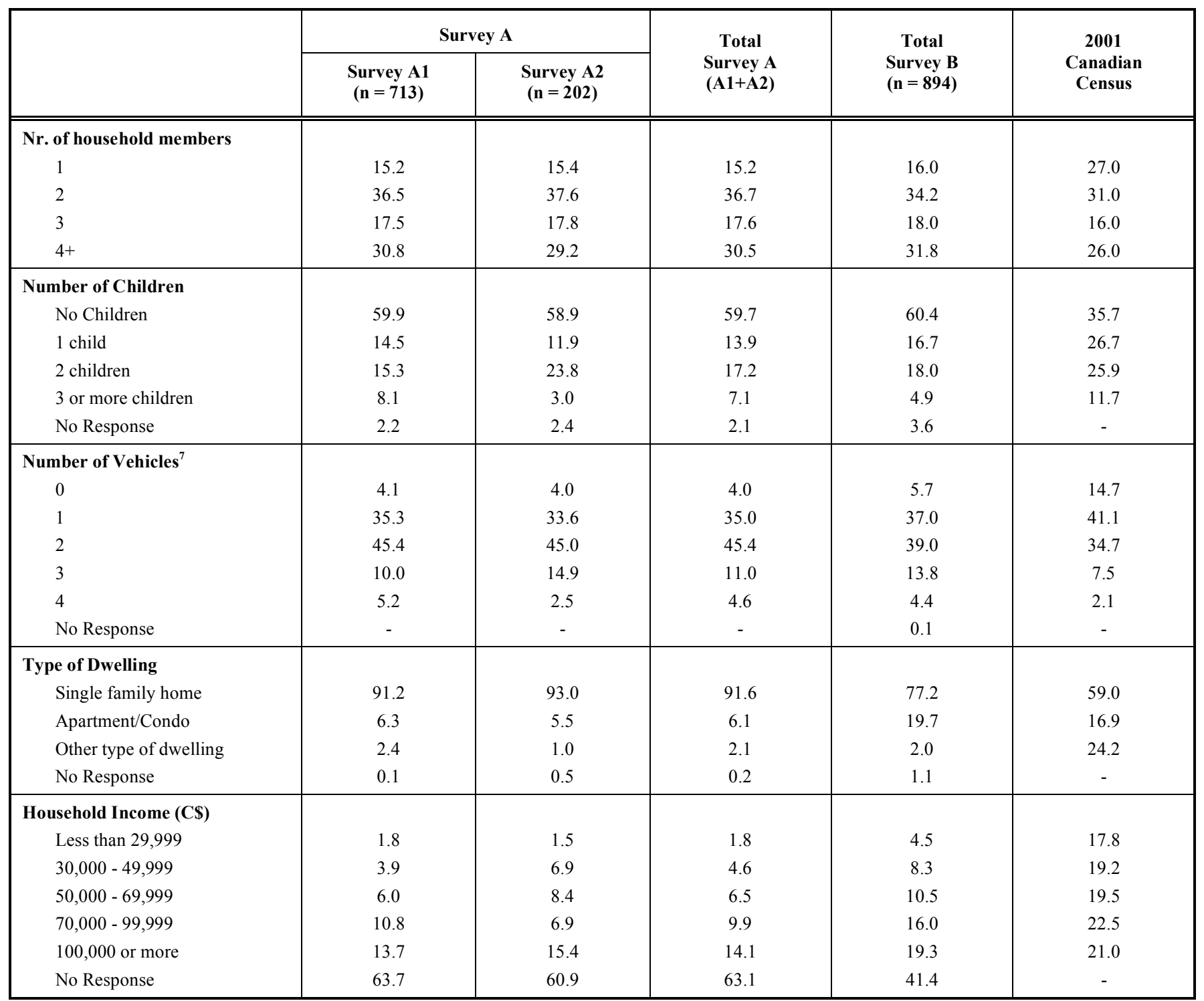

Moreover, concerns about measurement errors may be overcome through Internet surveys. While Internet-based surveys require careful design and thorough testing to maximize data quality, they generally appear more promising than telephone interviews. With regard to measurement errors, the most notable advantage of Internetbased surveys is that responses can be automatically saved into electronic databases, which reduces the risk of human errors in data entry and coding.

Non-response bias is generally higher when respondents are recruited via probability sampling. Comparing the proportions of non-response for several variables in the Internet samples collected using different recruitment methods, we find these proportions to be significantly different from each other. This finding implies that nonresponse is independent of the survey medium, but it is the recruitment method and sampling that affect it. This finding is not in line with the study by Fricker et al. [23] and de

${ }^{7}$ Compared against a representative sample of the 2001 Transportation Tomorrow Survey in the CMA of Hamilton
Blaeij et al. [26] who find no difference in item nonresponse between the Internet and telephone and Internet and mail surveys, respectively.

Examination of demographic characteristics across samples shows that younger individuals are over-represented and there is small percentage of older participants in the Internet samples. These findings agree with Sexton et al. [16] and the Pew Internet and American Life Project [17], but are contrary to de Blaeij et al. [26] who report that the elderly are more likely to participate in an Internet survey. However, there are no notable differences in the proportions of demographic variables of individuals between telephone and Internet-based responses when recruitment is conducted by probability sampling. This implies that it may be the sampling and recruitment methods that affect non-response bias rather than issues relating to technology challenges among respondents.

Finally, this paper adds to the body of evidence regarding the appropriateness of the Internet as a medium to collect survey data. Its findings highlight a number of issues that 
should be taken into consideration. The paper further points to the effectiveness of applying each of the mediums for survey purposes and how that is affected by communications, computer infrastructure, costs of communications and social and cultural aspects of the targeted populations. It is worth stressing that findings may have been different had the sampling method, unit of analysis (e.g. individual), geographical scale had been different. However, this paper provides significant evidence towards building the required body of knowledge for conducting a comprehensive metaanalysis of such studies, which in turn would offer robust generalisation regarding Internet survey design, implementation and quality assessment of data.

Table 5. Comparison of Internet and Telephone Surveys Based on the Empirical Analysis

\begin{tabular}{|l|c|c|c|}
\hline \multirow{2}{*}{} & $\begin{array}{c}\text { Convenience } \\
\text { Sampling }\end{array}$ & \multicolumn{2}{|c|}{$\begin{array}{c}\text { Probability } \\
\text { Sampling }\end{array}$} \\
\cline { 2 - 4 } & Internet & Phone & Internet \\
\hline \hline Response Speed & High & Low & Very Low \\
\hline Response Rates & Not Applicable & Low & Very Low \\
\hline Labour Requirements & Very Low & High & Low \\
\hline Expertise to Construct & High & Medium & High \\
\hline Measurement Error & Low & Medium & Low \\
\hline Coverage Error & High & Low & Medium \\
\hline Non-Response Error & High & Medium & Medium \\
\hline
\end{tabular}

\section{ACKNOWLEDGEMENTS}

The authors are grateful to the support of the City of Hamilton, the "Alexander S. Onassis" Benefit Foundation, the Social Sciences and Humanities Research Council (SSHRC) and the Canada Research Chairs program. In addition, the authors would like to thank Chris Benoit, Dorris McGuire, Deane Maynard, Laura Labate for their efforts on survey development and data collection. We are also grateful to Antonio Paez, Darren Scott, Thomas Adler, Chris Kavalec, Kara Kockelman, Heather Cotrino, Joachim Krapels and Jiri Skoupy for their comments and suggestions. Last but not least, the authors would like to thank the two anonymous referees for their constructive comments and suggestions.

\section{CONFLICT OF INTEREST}

Declared none.

\section{REFERENCES}

[1] R. Elmore-Yalch, J. Busby and C. Britton, Khow thy customer? Know thy research! A comparison of web-based and telephone responses to a public service customer satisfaction survey, 2007 Transportation Research Board 87 Annual Meeting, 2007.

[2] J. Zmud, "Designing Instruments to Improve Response," in Transport Survey Quality and Innovation, P. Stopher and P. Jones, Eds. Elsevier Science Ltd: Oxford, 2003.

[3] R. M. Groves and M. P. Couper, Nonresponse in Household Interview Surveys. Wiley: New York, 1998.

[4] A. Kellerman, "Internet access and penetration: An international urban comparison", J. Urban Tech., vol. 11, pp. 63-85, 2004.

[5] A. Loof, "Internet usage in 2008 - Households and individuals", 2008, Reference Available from: http://epp.eurostat.ec.europa.eu/
cache/ITY_OFFPUB/KS-QA-08-046/EN/KS-QA-08-046-EN.PDF [Accessed July, 2008].

[6] A. Collins, J. Rose and S. Hess, Search based internet surveys: Airline stated choice, 88th Annual Meeting Transportation Research Board, 2009.

[7] D. A. Dillman, Mail and Internet Surveys: The Tailored Design method. John Willey \& Sons: New York, 2000.

[8] H. F. Gunn, "Web-Based Surveys: Changing the Survey Process", 2002, Reference Available from: http://firstmonday.org/htbin/cgi wrap/bin/ojs/index.php/fm/article/view/1014/935. [Accessed Nov. 09, 2011].

[9] T. Whelan, Anecendents of Anonymity Perceptions in Web-Based Surveys, 23rd Annual Meeting of the Society of Industrial Organisational Psychology, 2008.

[10] K. Braunsberger, H. Wybenga and R. Gates, "A comparison of reliability between telephone and web-based survey", J. Bus. Res., vol. 60, pp. 758-764, 2007.

[11] W. L. Richman, S. Kiesler, S. Weisband and F. Drasgow, "A metaanalytic study of social desirability distortion in computeradministered questionnaires, traditional questionnaires, and interviews.", J. Appl. Psychol., vol. 84, pp. 754-775, 1999.

[12] E. Deutskens, A. d. Jong and K. d. Ruyter, "Comparing the generalizability of online and mail surveys and cross-national service quality research", Marketing Sci., vol. 17, pp. 119-136, 2006.

[13] Y. Zhang, "Using the Internet for survey research: A case study", $J$. Am. Soc. Inf. Sci., vol. 51, pp. 57-68, 1999.

[14] M. Demoussis and N. Giannakopoulos, "Facets of the digital divide in Europe: Determination and extent of Internet use", Econ. Innovation New Tech., vol. 15, pp. 235-246, 2006.

[15] S. Sills and C. Song, "Innovations in survey research", Soc. Sci. Comp. Rev., vol. 20, pp. 22-30, 2002.

[16] N. Sexton, H. M. Miller and A. M. Dietsch, "Appropriate uses and considerations for online surveying in human dimensions research", Hum. Dim. Wildlife, vol. 16, pp. 154-163, 2011.

[17] Pew Internet \& American Life Project, "Demographics of Internet Users", 2010, Reference Available from: http://pewinternet.org/ Trend-Data/Whos-Online.aspx [Accessed 24 October 2011].

[18] C. Parsons, "Web-based surveys: Best practices based on the research literature", Visitor Stud., vol. 10, pp. 13-33, 2007.

[19] AAPOR. (2008, Oct. 2009). AAPOR Report on online panels. Available: http://www.aapor.org/AM/Template.cfm?Section=AAP OR_Committee_and_Task_Force_Reports\&Template $=/ \mathrm{CM} / \mathrm{Conte}$ ntDisplay.cfm\&ContentID $=2223$

[20] A. D. Dillman and D. Smyth, "Design effects in the transition to web-based surveys", Am. J. Prev. Med., vol. 32, pp. S90-S96, 2007.

[21] C. Cobanoglu, B. Warde and P. J. Moreo, "A comparison of mail, fax and web-based survey methods", Int. J. Market Res., vol. 43, pp. 441-452, 2001

[22] S. Crawford, S. McCabe and C. Boyd, Improving response rates and data collection efficiencies. International conference on improving surveys, International Conference on Improving Surveys, 2002.

[23] S. Fricker, M. Galesic, R. Tourangeai and T. Yan, "An experimental comparison of web telephone surveys", Public Opin. Q., vol. 69, pp. 370-392, 2005.

[24] T. Arentze, I. Bos, E. Molin and H. Timmermans, "Internet-based travel surveys: Selected evidence on response rates, sampling bias and reliability", Transportmetrica, vol. 1, pp. 193-207, 2005.

[25] C. Bayart and P. Bonnel, Comparison of web and face-to-face household travel survey - application to Lyon case, 2008 European Transport Conference, 2008.

[26] T. A. De Blaeij, M. Dijst and S. Farag, Effects of data collection methods in travel behaviour surveys: Comparing an internet and a mail sample, Transportation Research Board 2006 Annual Meeting - CD-ROM, 2006.

[27] W. Mix, D. Chernoff and T. Trabold, A comparison of internet and mail-based transportation surveys, 2008 Transportation Research Board 87th Annual Meeting, 2008.

[28] D. Potoglou, "Demand for Conventional and Alternative Fuelled Vehicles in the Census Metropolitan Area of Hamilton, Canada", Ph.D. thesis, McMaster University, Hamilton, ON, Canada, 2006.

[29] Fuel Economy Guide, "Fuel Economy Data Download", 19722005, Reference Available from: http://www.fueleconomy.gov/feg/ download.shtml [Accessed Aug. 11, 2009]. 
[30] D. Potoglou and P. Kanaroglou, "An Internet-Based Stated Choice Household Survey for Alternative Fuelled Vehicles", Can. J. Transp., vol. 1, pp. 36-55, 2007.

[31] P. A. Champ and M. P. Welsh, "Survey methodologies for stated choice studies," in Valuing Environmental Amenities Using Stated Choice Studies, B. J. Kanninen, Ed. Springer: Heidelberg, 2006, pp. 21-42.

[32] M. Schonlau, R. M. Fricker and M. N. Elliott, Conducting Research Surveys via E-mail and the Web, RAND Corporation,
Santa Monica MR-1480-RC, 2002. Available from: http://www. rand.org/pubs/monograph_reports/MR1480.html

[33] P. Iraguen and J. Ortuzar, "Willingness-to-pay for reducing fatal accident risk in urban areas: an Internet-based Web page stated preference survey", Accid. Anal. Prev., vol. 36, pp. 513-524, 2004.

[34] M. P. Couper, A. Kapteyn, M. Schonlau and J. Winter, "Noncoverage and nonresponse in an Internet survey", Soc. Sci. Res., vol. 36, pp. 131-148, 2007.

Received: December 202011

Revised: December 31, 2011

Accepted: January 2, 2012

(C) Potoglou et al.; Licensee Bentham Open.

This is an open access article licensed under the terms of the Creative Commons Attribution Non-Commercial License (http://creativecommons.org/licenses/by-nc/

3.0/) which permits unrestricted, non-commercial use, distribution and reproduction in any medium, provided the work is properly cited. 